\title{
Atomic parity violation in cesium and implications for the 3 - 3 - 1 models
}

\author{
Hoang Ngoc Long ${ }^{\mathrm{a} *}$ and Le Phuoc Trung ${ }^{\mathrm{b}}$ \\ ${ }^{a}$ The Abdus Salam International Centre for Theoretical Physics, Trieste, Italy \\ ${ }^{\mathrm{b}}$ HCMC Institute of Physics, Mac Dinh Chi 1, Ho Chi Minh city, Vietnam
}

The parity violation in cesium atom is analysed in the framework of the models based on the $\mathrm{SU}(3)_{C} \times \mathrm{SU}(3)_{L} \times$ $\mathrm{U}(1)_{N}$ gauge group. It is shown that in the minimal version, the main contribution to a deviation of weak charge $\Delta Q_{W}$ due to direct $Z^{\prime}$ exchange is negative. New data on parity violation in the cesium atom seems not favour to the minimal version, while it gets a positive value in the version with right-handed neutrinos. We obtain a bound on the $Z^{\prime}$ mass at a range from $1.4 \mathrm{TeV}$ to $2.6 \mathrm{TeV}$. The allowed regions for the $Z-Z^{\prime}$ mixing angle are also derived.

PACS number(s): 11.30.Er,12.15.Ji,12.15.Mm,12.60.Cn

\section{Introduction}

One prediction of the standard model (SM) is the existence of the atomic parity violation (APV). The new data on the APV in cesium atom [1] has caused extensive interest and reviews [2]. Parity violation in the SM results from exchanges of weak gauge bosons. In electronhadron neutral-current processes parity violation is due to the vector axial-vector interaction in the Lagrangian. The measurement is stated in terms of the weak charge $Q_{W}$, which parameterizes the parity violating Lagrangian. The value reported

$Q_{W}\left({ }_{55}^{133} \mathrm{Cs}\right)=-72.06 \pm 0.28$ (expt) \pm 0.34 (theo) $(1)$

represents a considerable improvement over the previous determination [3]. Compared to the SM prediction $Q_{W}^{S M}$, the deviation $\Delta Q_{W}$ is

$\Delta Q_{W} \equiv Q_{W}(\mathrm{Cs})-Q_{W}^{\mathrm{SM}}(\mathrm{Cs})=1.03 \pm 0.44$,

which is $2.3 \sigma$ away from the SM prediction. This value has been widely used for analysis of possible new physics.

Among the beyond SM extensions, the recent proposed models based on the $\mathrm{SU}(3)_{C} \times \mathrm{SU}(3)_{L} \times$ $\mathrm{U}(1)_{N}$ gauge group 47 (hereafter 3 - 3 - 1 models) have the following intriguing features: firstly,

\footnotetext{
*On leave from Institute of Physics, NCST, P.O.Box 429, Bo Ho, Hanoi 10000, Vietnam. E-mail address: hnlong@iop.ncst.ac.vn
}

the models are anomaly free only if the number of families $N$ is a multiple of three. Further, from the condition of QCD asymptotic freedom, which means $N<5$, it follows that $N$ is equal to 3 . The second characteristic is that the Lagrangians of these models possess the Peccei-Quinn symmetry naturally, hence the strong $C P$ problem can be solved in an elegant way [8]. The third interesting feature is that one of the quark families is treated differently from the other two. This could lead to a natural explanation of the unbalancing heavy top quark in the fermion mass hierarchy.

There are two main versions of the $3-3$ 1 models: the minimal model in which all lepton components $\left(\nu, l, l^{c}\right)_{L}$ of each family belong to one and same lepton triplet and a variant, in which right-handed $(\mathrm{RH})$ neutrinos are included, i.e. $\left(\nu, l, \nu^{c}\right)_{L}$ (hereafter we call it the model with right-handed neutrinos [6]7]). New gauge bosons in the minimal model are bileptons $\left(Y^{ \pm}, X^{ \pm \pm}\right)$carrying lepton number $L= \pm 2$ and a new $Z^{\prime}$. In the second model, the bileptons with lepton number $L= \pm 2$ are singly-charged $Y^{ \pm}$and neutral gauge bosons $X^{0}, X^{* 0}$, and both are responsible for lepton-number violating interactions. Thus, with the present group extension there are five new gauge bosons and all these particles are heavy. Getting mass limits for these particles is one of the central tasks of further stud- 
Table 1

Vector and axial-vector coupling constants relevant for APV in cesium atom for the SM and for two 3 3 - 1 models

\begin{tabular}{|c|c|c|}
\hline Standard model & $3-3-1$ model with RH neutrinos & Minimal 3 - 3 - 1 model \\
\hline \hline$a_{e}=\frac{1}{2}$ & $a_{e}^{\prime}=-\frac{1}{2} \frac{1}{\sqrt{3-4 s_{W}^{2}}}$ & $a_{e}^{\prime}=\frac{\sqrt{1-4 s_{W}^{2}}}{2 \sqrt{3}}$ \\
$v_{u}=\frac{1}{2}-\frac{4 s_{W}^{2}}{3}$ & $v_{u}^{\prime}=-\left(\frac{1}{2}-\frac{4 s_{W}^{2}}{3}\right) \frac{1}{\sqrt{3-4 s_{W}^{2}}}$ & $v_{u}^{\prime}=\frac{1}{2 \sqrt{3}} \frac{\left(-1+6 s_{W}^{2}\right.}{\sqrt{1-4 s_{W}^{2}}}$ \\
$v_{d}=-\frac{1}{2}+\frac{2 s_{W}^{2}}{3}$ & $v_{d}^{\prime}=-\left(\frac{1}{2}-\frac{s_{W}^{2}}{3}\right) \frac{1}{\sqrt{3-4 s_{W}^{2}}}$ & $v_{d}^{\prime}=-\frac{1}{2 \sqrt{3}} \frac{1}{\sqrt{1-4 s_{W}^{2}}}$ \\
\hline
\end{tabular}

ies.

We notice that it is rather easy to get bounds on masses of the bileptons following from, for example, the "wrong" muon decay data, from the anomalous magnetic moments of the muon [9] or from radiative corrections 10,11. New neutral gauge boson beyond the photon and the $Z$ of the SM has long been considered as one of the most interesting topics. However, getting bounds on mass of the $Z^{\prime}$ in these models is rather difficult. Perhaps the APV is one of the most suitable subjects for this purpose. The aim of this paper is to consider the atomic parity violation in the 3 - 3 1 models and imply it to get bounds on mass of the $Z^{\prime}$ boson.

\section{Atomic parity violation in the minimal 3 - 3 - 1 model}

Basic to the analysis of the experiments on parity violation in atoms is the electron-quark effective Lagrangian

$\mathcal{L}_{e f f}=\frac{G_{F}}{\sqrt{2}}\left(\bar{e} \gamma_{\mu} \gamma_{5} e\right)\left(C_{1 u} \bar{u} \gamma_{\mu} u+C_{1 d} \bar{d} \gamma_{\mu} d\right)$.

We see that it involves only electron coupling being axial (or spin dependent) and quark coupling vector. This interaction between the electron and the nucleus is thus coherent, proportional to the total weak charge. There is a similar interaction which is also generated by direct $Z$ exchange, but with the electron coupling being vector and the nuclear coupling axial vector. This kind of interaction is inhibited: the axial coupling to the nucleus is no longer coherent (only the last unpaired nucleon contributes), and the electron vector coupling is quite suppressed $(\sim$ $0.026)$. In addition, one knows that the electromagnetic interaction of atomic electrons with the nuclear anapole moment may generate a measurable spin dependence in atomic parity violation experiments. This interaction, which is considerably smaller than the coherent $Z$ interaction, may exceed the direct nuclear spin dependent parity violation for heavy atoms [12]. Hence one may neglect the nuclear spin dependent parity violations.

The experimental results are usually quoted in terms of the related so-called weak charge

$Q_{W}=-2\left[C_{1 u}(2 Z+N)+C_{1 d}(Z+2 N)\right]$,

where $Z$ and $N$ are the number of protons and neutrons, respectively, of the atom considered. We are interested in the deviation $\Delta Q_{W}=Q_{W}-$ $Q_{W}^{S M}$ with respect to the SM predictions. This deviation is given as 13 .

$$
\begin{aligned}
\Delta Q_{W}= & {\left[\left(1+4 \frac{s_{W}^{4}}{\cos 2 \theta_{W}}\right) Z-N\right] \Delta \rho_{M} } \\
& +16\left[(2 Z+N)\left(a_{e} v_{u}^{\prime}+a_{e}^{\prime} v_{u}\right)\right. \\
& \left.+(Z+2 N)\left(a_{e} v_{d}^{\prime}+a_{e}^{\prime} v_{d}\right)\right] \phi \\
& +16\left[(2 Z+N) a_{e}^{\prime} v_{u}^{\prime}\right. \\
& \left.+(Z+2 N) a_{e}^{\prime} v_{d}^{\prime}\right] \frac{m_{Z}^{2}}{M_{Z^{\prime}}^{2}},
\end{aligned}
$$

where $\phi$ is the mixing angle between the new gauge boson $Z^{\prime}$ and the canonical $Z, v_{f}, a_{f}, v_{f}^{\prime}$, $a_{f}^{\prime}$ are couplings of $Z, Z^{\prime}$ to fermions. The couplings are defined in the neutral interactions

$$
\mathcal{L}(Z)=\frac{g}{2 \cos \theta_{W}} \sum_{f}\left(v_{f} \bar{f} \gamma_{\mu} f+a_{f} \bar{f} \gamma_{\mu} \gamma_{5} f\right) Z^{\mu},
$$




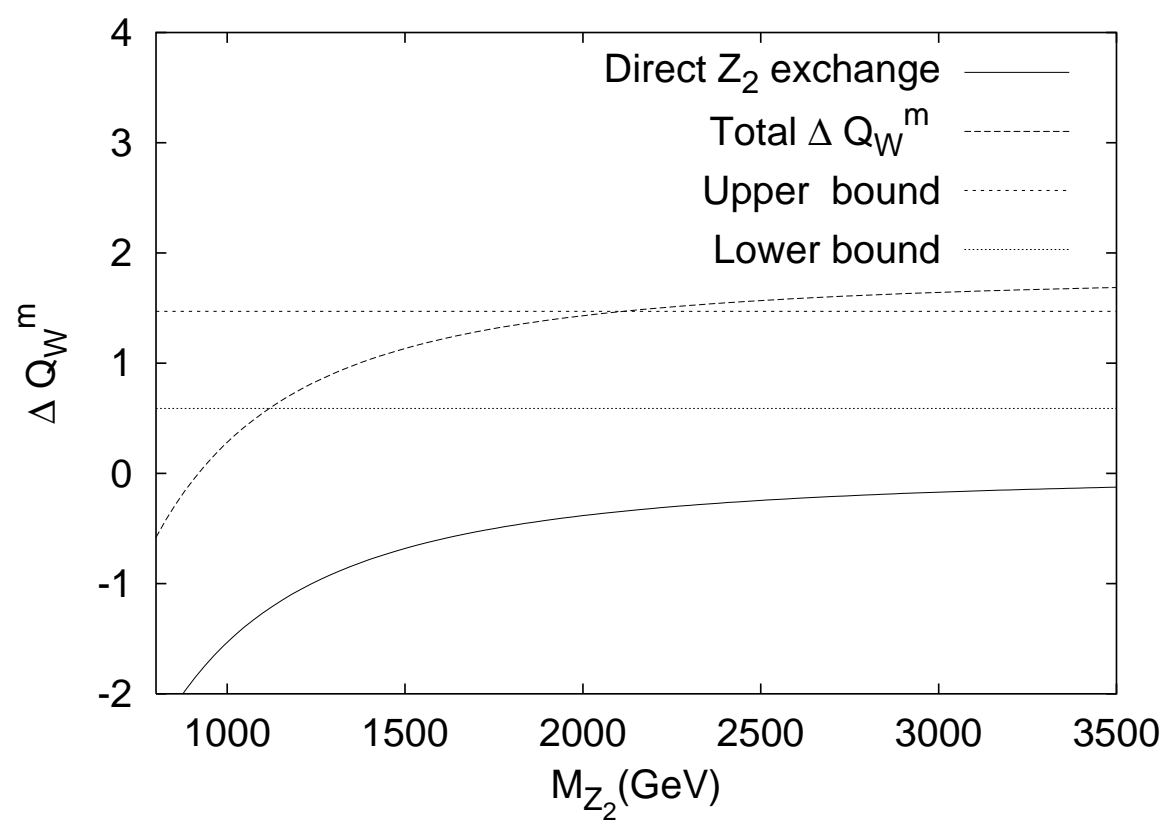

Figure 1. $\Delta Q_{W}^{m}$ as a function of $M_{Z^{\prime}}$. The continuous line is obtained by neglecting the contribution from the $Z-Z^{\prime}$ mixing.

$\mathcal{L}\left(Z^{\prime}\right)=\frac{g}{2 \cos \theta_{W}} \sum_{f}\left(v_{f}^{\prime} \bar{f} \gamma_{\mu} f+a_{f}^{\prime} \bar{f} \gamma_{\mu} \gamma_{5} f\right) Z^{\prime \mu}$

where the summation is over all relevant fermions. The coupling constants relevant for calculations of APV in the cesium atom for the SM, 3 - 3 1 model with RH neutrinos and the minimal version are presented in Table 1, in which the usual notation is used: $s_{W} \equiv \sin \theta_{W}$. More details on the 3 - 3 - 1 models can be found in Refs. [4, 6, 14].

We notice that the first term on the righthanded side of Eq. (5) is proportional to $\Delta \rho_{M}$, which is an additional contribution to the $\rho$ parameter arising from the mixing. This term receives two independent contributions. The first one comes from the overall $\rho$ factor on the righthanded side of Eq. (2.15) in 13. The second one arises from a shift in $\sin ^{2} \theta_{W}$, which is proportional to $\Delta \rho_{M}$. The second term is due to the $Z-Z^{\prime}$ mixing. These two terms are ultimately dependent upon the mixing angle $\phi$ and particle content. The third term, which is proportional to the squared mass ratio $m_{Z}^{2} / M_{Z^{\prime}}^{2}$, is due to the direct $Z^{\prime}$ exchange.

In the 3 - 3 - 1 models, deviation of the parameter $\Delta \rho_{M}$ gets contributions from both oblique corrections and the $Z-Z^{\prime}$ mixing, and it relates to the oblique radiative parameter $T$ [15] as the following

$\Delta \rho_{M}=\alpha T$.

In the minimal model, it was shown that [10,11

$$
\begin{aligned}
T^{m}= & \frac{3 \sqrt{2} G_{F}}{16 \pi^{2} \alpha}\left[M_{++}^{2}+M_{+}^{2}\right. \\
& \left.-\frac{2 M_{++}^{2} M_{+}^{2}}{M_{++}^{2}-M_{+}^{2}} \ln \frac{M_{++}^{2}}{M_{+}^{2}}\right] \\
& +\frac{1}{4 \pi s_{W}^{2}}\left[2 \bar{F}_{0}\left(0, M_{++}, M_{+}\right)\right. \\
& \left.+3 t_{W}^{2} \ln \frac{M_{++}^{2}}{M_{+}^{2}}\right] \\
& +\frac{\tan ^{2} \phi}{\alpha}\left(\frac{M_{Z^{\prime}}^{2}}{m_{Z}^{2}}-1\right)
\end{aligned}
$$




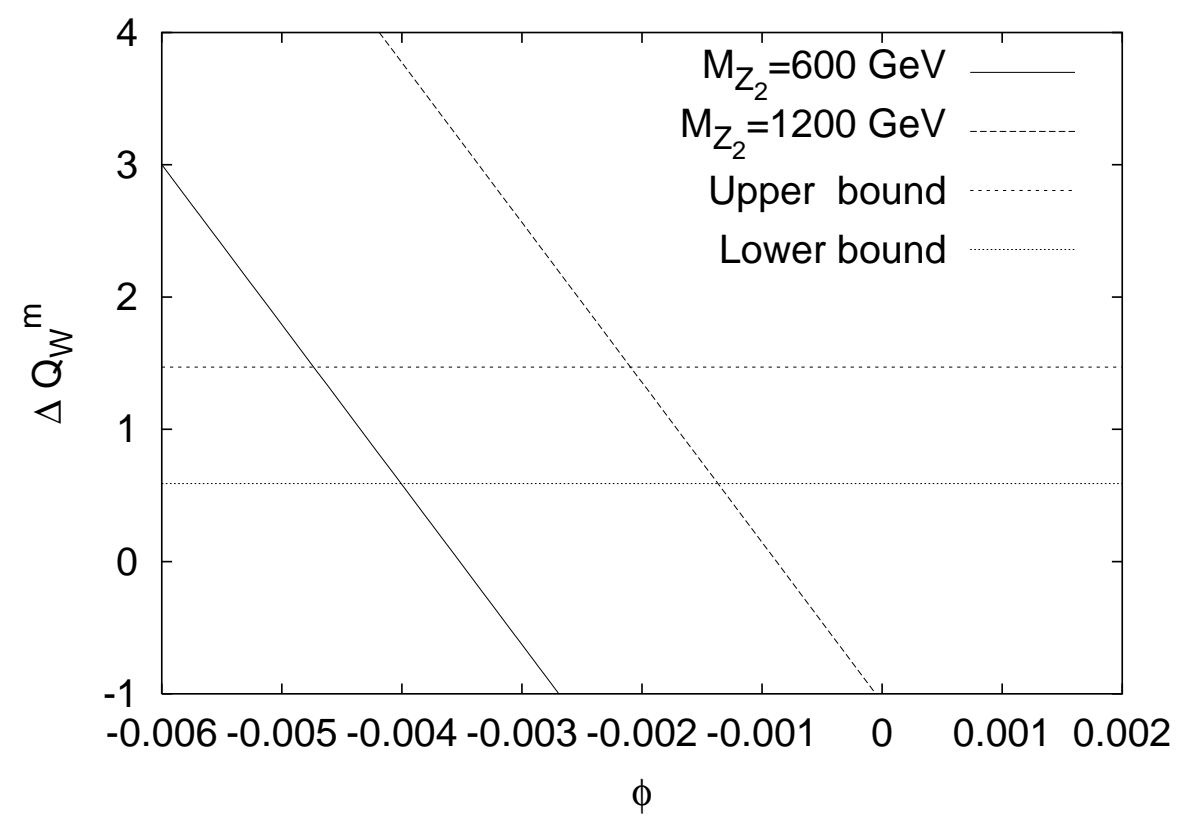

Figure 2. $\Delta Q_{W}^{m}$ as a function of $\phi$. The continuous line refers to $M_{Z^{\prime}}=600 \mathrm{GeV}$, the dashed line $1200 \mathrm{GeV}$.

where $\bar{F}_{0}(s, M, m) \equiv \int_{0}^{1} d x \ln \left[(1-x) M^{2}+x m^{2}-\right.$ $x(1-x) s]-\ln M m$, and $M_{+}, M_{++}$stand for masses of the singly- and doubly-charged gauge bosons $Y^{+}, X^{++}$, respectively. The first two terms in (7) are polynomial in the ratio between mass splitting and the bilepton mass [11]: $\varepsilon \equiv$ $\varepsilon\left(M_{+}, M_{++}\right)=\frac{M_{+}^{2}-M_{++}^{2}}{M_{++}^{2}}$. The superscript $m$ in $T^{m}$ refers to the parameter in the minimal version.

By the spontaneous symmetry breaking, the bilepton mass splitting is given (see N. A. Ky et al in [9])

$\left|M_{++}^{2}-M_{+}^{2}\right| \leq 3 m_{W}^{2}$.

Therefore the parameter $\varepsilon$ is bounded too. This leads to limitation in the $T$ parameter.

With the $Z^{\prime}$ mass in a range of $1 \mathrm{TeV}$ scale, the last term in (7) gives just contribution of about $4 \%$ to the $T^{m}$ parameter 11$]$.

The $Z-Z^{\prime}$ mixing angle $\phi$ is constrained 14: $-5 \times 10^{-3} \leq \phi \leq 7 \times 10^{-4}$, hence the two last terms in (5) give a dominant contribution.

Using the following data [16]: $s_{W}^{2}=$ $0.23117, m_{Z}=91.1882 \mathrm{GeV}, M_{+}=$ $230 \mathrm{GeV}, M_{++}=300 \mathrm{GeV}$, we obtain

$\Delta Q_{W}^{m} \simeq-0.01-1210.07 \phi-184.32\left(\frac{m_{Z}^{2}}{M_{Z^{\prime}}^{2}}\right)$.

In Fig. 1 we plot the direct $Z^{\prime}$ exchange contribution (the continuous line) and $\Delta Q_{W}^{m}$ (the dashed line) as a function of $M_{Z^{\prime}}$. We see that the direct $Z^{\prime}$ exchange contribution is negative. The coefficient of $\phi$ in the second term - the $Z-Z^{\prime}$ mixing contribution is negative too. Therefore the total $\Delta Q_{W}^{m}$ get a negative value if $\phi$ is positive. This circumstance is excluded at 99\% CL (see R. Casalbuoni et al. in |2|). The dashed line corresponds to the total $\Delta Q_{W}^{m}$ at the $\phi=-1.5 \times 10^{-3}$. In this case the constraint for the $Z^{\prime}$ mass is $1.15 \mathrm{TeV} \leq M_{Z^{\prime}} \leq 2.2 \mathrm{TeV}$.

In Fig. 2 we plot $\Delta Q_{W}^{m}$ as a function of the $Z-Z^{\prime}$ mixing angle $\phi$ at two values for $M_{Z^{\prime}}=600$ 


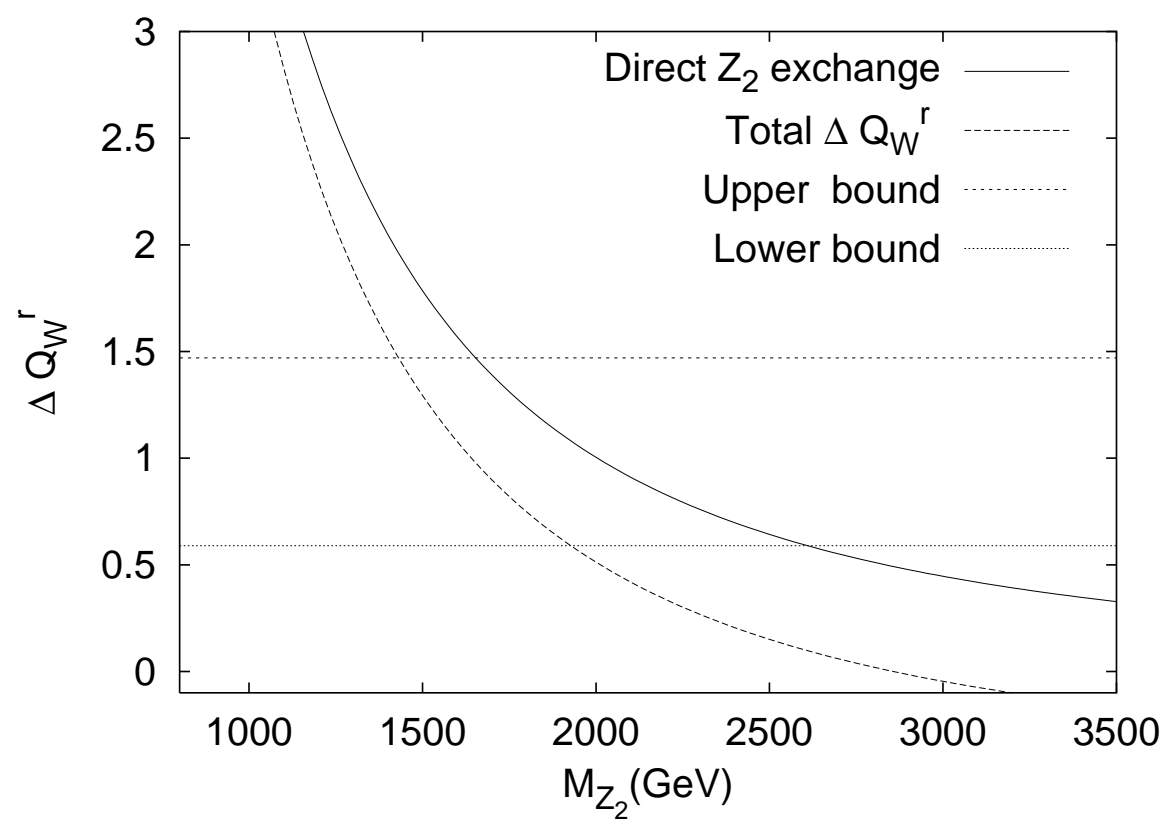

Figure 3. $\Delta Q_{W}^{r}$ as a function of $M_{Z^{\prime}}$. The continuous line is obtained by neglecting the contribution from the $Z-Z^{\prime}$ mixing.

$\mathrm{GeV}$ (the dashed line) and $M_{Z^{\prime}}=1200 \mathrm{GeV}$ (the continuous line). However we can make the $\Delta Q_{W}^{m}$ positive by taking $\phi$ is negative and large enough, say in order of few $\times 10^{-3}$. In conclusion, to be consistent with the new data on parity violation in the cesium atom, the $Z-Z^{\prime}$ mixing angle $\phi$ in the minimal $3-3-1$ model has to be negative and is in order of a few $\times 10^{-3}$

$$
\begin{aligned}
-0.0045 & \leq \phi \leq-0.004 \text { for } M_{Z^{\prime}}=600 \mathrm{GeV} \\
-0.002 & \leq \phi \leq-0.0018 \text { for } M_{Z^{\prime}}=1200 \mathrm{GeV}
\end{aligned}
$$

\section{Atomic parity violation in the $3-3-1$ model with RH neutrinos}

In the model with $\mathrm{RH}$ neutrinos the bileptons $Y^{+}, X^{0}$ make an $S U(2)_{L}$ doublet with hypercharge $Y=\frac{1}{2}$. The $T$ parameter in this model is given as [1]

$T^{r}=\frac{3 \sqrt{2} G_{F}}{16 \pi^{2} \alpha}\left[M_{+}^{2}+M_{0}^{2}-\frac{2 M_{+}^{2} M_{0}^{2}}{M_{+}^{2}-M_{0}^{2}} \ln \frac{M_{+}^{2}}{M_{0}^{2}}\right]$

$$
\begin{aligned}
& +\frac{1}{4 \pi s_{W}^{2}}\left[2 \bar{F}_{0}\left(0, M_{+}, M_{0}\right)+t_{W}^{2} \ln \frac{M_{+}^{2}}{M_{0}^{2}}\right] \\
& +\frac{\tan ^{2} \phi}{\alpha}\left(\frac{M_{Z^{\prime}}^{2}}{m_{Z}^{2}}-1\right),
\end{aligned}
$$

where $M_{+}, M_{0}$ stand for masses of the singlycharged and neutral gauge bosons $Y^{+}, X^{0}$, respectively. Again, the first two terms in (10) are polynomial in $\epsilon \equiv \epsilon\left(M_{+}, M_{0}\right)=\frac{M_{+}^{2}-M_{0}^{2}}{M_{0}^{2}}$.

As before, the spontaneous symmetry breaking gives the bilepton mass splitting 11

$\left|M_{0}^{2}-M_{+}^{2}\right| \leq m_{W}^{2}$.

Hence the parameter $\epsilon$ is bounded shorter than one in the minimal version. The contribution from the first term in (5) is negligible.

The $Z-Z^{\prime}$ mixing angle $\phi$ is also constrained to be very small $-2 \times 10^{-4} \leq \phi \leq 3 \times 10^{-3}$, therefore the last term in (5) gives a dominant contribution. 


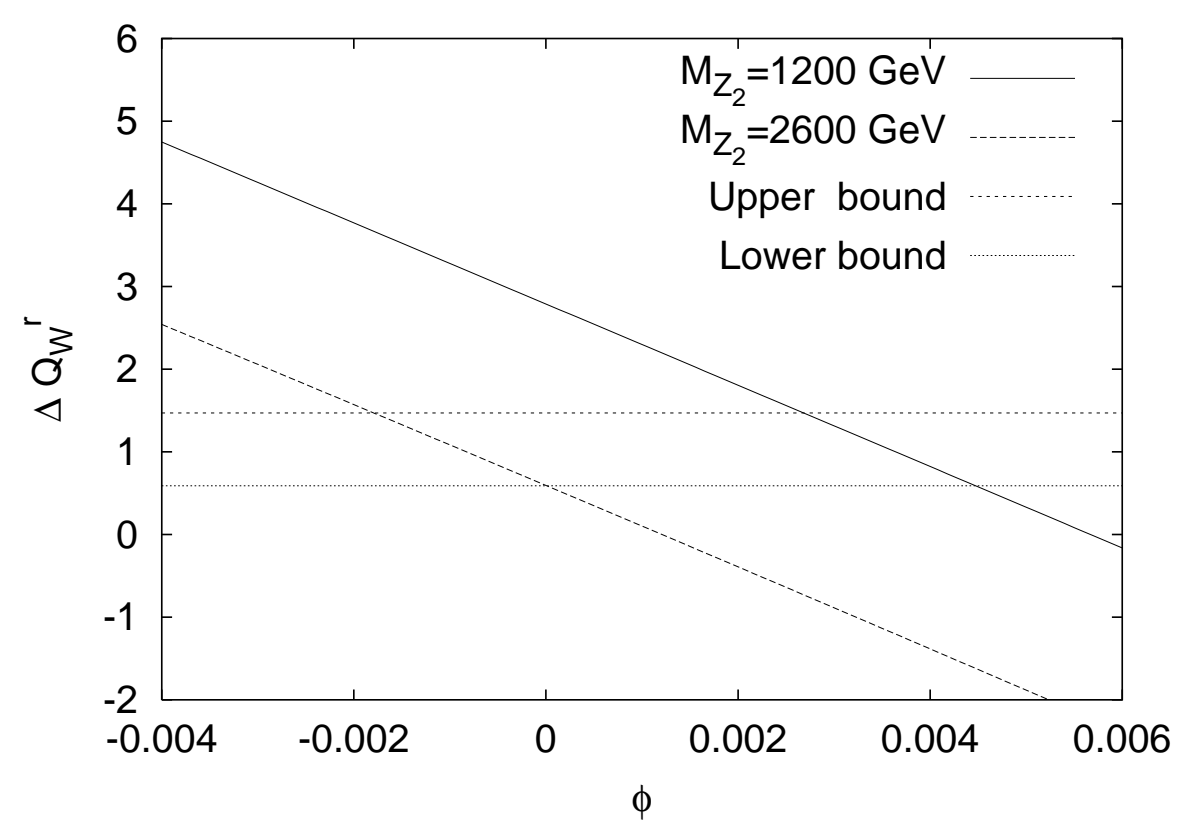

Figure 4. $\Delta Q_{W}^{r}$ as a function of $\phi$. The continuous line refers to $M_{Z^{\prime}}=1200 \mathrm{GeV}$, the dashed line $2600 \mathrm{GeV}$.

Taking into account that 11: $M_{+}=$ $230 \mathrm{GeV}, M_{0}=250 \mathrm{GeV}$, we get

$\Delta Q_{W}^{r} \simeq-0.00092-490.71 \phi+482.99\left(\frac{m_{Z}^{2}}{M_{Z^{\prime}}^{2}}\right)$.

Here the sign of the last term is plus. The coefficient of $\phi$ in the second term of $\Delta Q_{W}^{r}$, where $r$ refers to the model with $\mathrm{RH}$ neutrinos, is negative and is much smaller than those in the minimal version.

In Fig. 3 we plot the direct $Z^{\prime}$ exchange contribution (the continuous line) and $\Delta Q_{W}^{r}$ (the dashed line) as a function of $M_{Z^{\prime}}$. Here we take the $Z-Z^{\prime}$ mixing angle $\phi=0.001$. The contribution of the second term is negative if the mixing angle $\phi$ is positive. However it is just $10 \%$ of total value. From the figure we get the constraint for the $Z^{\prime}$ mass: $1.4 \mathrm{TeV} \leq M_{Z^{\prime}} \leq 1.9 \mathrm{TeV}$ in the case of the total $\Delta Q_{W}^{r}$. This number is slightly higher: $1.7 \mathrm{TeV} \leq M_{Z^{\prime}} \leq 2.6 \mathrm{TeV}$ in the case of neglecting the $Z-Z^{\prime}$ mixing.
In Fig. 4 we plot $\Delta Q_{W}^{r}$ as a function of the $Z-Z^{\prime}$ mixing angle $\phi$ at two values for $M_{Z^{\prime}}=$ $1200 \mathrm{GeV}$ (the dashed line) and $M_{Z^{\prime}}=2600 \mathrm{GeV}$ (the continuous line). From the figure it follows that an allowed region for the mixing angle is

$$
\begin{aligned}
0.0028 & \leq \phi \leq 0.0046 \text { for } M_{Z^{\prime}}=1200 \mathrm{GeV}, \\
-0.0018 & \leq \phi \leq 0 \text { for } M_{Z^{\prime}}=2600 \mathrm{GeV} .
\end{aligned}
$$

Summarizing this section, we conclude that the APV data provides a large room for the $3-3-1$ model with RH neutrinos.

\section{Conclusions}

We have considered parity violation in the cesium atom. It is shown that in the minimal version the main contribution to a deviation of weak charge $\Delta Q_{W}$ due to direct $Z^{\prime}$ exchange, is negative. To make the deviation positive, the $Z-Z^{\prime}$ mixing angle $\phi$ has to be negative and large enough, say in order of few $\times 10^{-3}$. In the case of $\phi=-0.0015$ the constraint for the $Z^{\prime}$ mass is 
$1.15 \mathrm{TeV} \leq M_{Z^{\prime}} \leq 2.2 \mathrm{TeV}$. New data on parity violation in the cesium atom seems not favour to the minimal version.

The deviation of weak charge in the $3-3-1$ model with $\mathrm{RH}$ neutrinos is positive and is mainly from the $Z^{\prime}$ exchange. For the mixing angle $\phi=0.001$ we get the constraint for the $Z^{\prime}$ mass: $1.4 \mathrm{TeV} \leq M_{Z^{\prime}} \leq 1.9 \mathrm{TeV}$ in the case of the total $\Delta Q_{W}^{r}$. Neglecting the $Z-Z^{\prime}$ mixing we get the constraint: $1.7 \mathrm{TeV} \leq M_{Z^{\prime}} \leq 2.6 \mathrm{TeV}$. If mass of the $Z^{\prime}$ lying around present experimental limit of about $600 \mathrm{GeV}$ [17], then the allowed region for the $Z-Z^{\prime}$ mixing angle in the minimal version is: $-0.0045 \leq \phi \leq-0.004$, which is comparable to limit extracted from the $Z$-decay experiments. For the model with RH neutrinos, in order to have a mixing angle consistent to that from the $Z$ decay data, i.e. $\phi \approx-2 \times 10^{-4} \div 3 \times 10^{-3}$, mass of the $Z^{\prime}$ has to be heavier than $1 \mathrm{TeV}$.

Finally, we mention that parity violation in the cesium atom provides a useful tool for getting bounds on mass of the new $Z^{\prime}$ and its mixing with the $\mathrm{Z}$ boson in the SM.

\section{Acknowledgments}

One of the authors (H. N. L.) would like to thank the Abdus Salam International Centre for Theoretical Physics, Trieste, Italy for financial support and hospitality. This work was supported in part by the Natural Science Council of Vietnam.

\section{REFERENCES}

1. S. C. Bennett, C. E. Wieman, Phys. Rev. Lett. 82 (1999) 2484.

2. R. Casalbuoni, S. De Curtis, D. Dominici and R. Gatto, Phys. Lett. B 460 (1999) 135 ; J. L Rosner, Phys. Rev. D 61 (2000) 016006; V. Barger and K. Cheung, Phys. Lett. B 480 (2000) 149; J. Erler and P. Langacker, Phys. Rev. Lett. 84 (2000) 212.

3. C. S. Wood, et al., Science 275 (1997) 1759.

4. F. Pisano and V. Pleitez, Phys. Rev. D 46 (1992) 410; P. H. Frampton, Phys. Rev. Lett. 69 (1992) 2889.

5. R. Foot, O.F. Hernandez, F. Pisano and V. Pleitez, Phys. Rev. D 47 (1993) 4158.

6. R. Foot, H. N. Long and Tuan A. Tran, Phys.
Rev. D 50 (1994) R34; H. N. Long, Phys. Rev. D 53 (1996) 437; Phys. Rev. D 54 (1996) 4691.

7. J. C. Montero, F. Pisano and V. Pleitez, Phys. Rev. D 47 (1993) 2918.

8. P. B. Pal, Phys. Rev. D 52 (1995) 1659.

9. H. Fujii, S. Nakamura and K. Sasaki, Phys. Lett. B 299 (1993) 342; N. A. Ky, H. N. Long and D. V. Soa, Phys. Lett. B 486 (2000) 140.

10. K. Sasaki, Phys. Lett. B 308 (1993) 297; P. H. Frampton and M. Harada, Phys. Rev. D 58 (1998) 09513.

11. H. N. Long and T. Inami, Phys. Rev. D 61 (2000) 075002.

12. W. C. Haxton, Science 275 (1997) 1753.

13. G. Altarelli, R. Casalbuoni, S. De Curtis, N. Di Bartolomeo, F. Feruglio and R. Gatto, Phys. Lett. B 261 (1991) 146.

14. D. Ng, Phys. Rev. D 49 (1994) 4805.

15. M. E. Peskin and T. Takeuchi, Phys. Rev. Lett. 65 (1990) 964; Phys. Rev, D 46 (1992) 381.

16. D. E. Groom et al., Particle Data Group, Eur. Phys. J. C 15 (2000) 1.

17. CDF Collaboration, F. Abe et al., Phys. Rev. Lett. 79 (1997) 2192. 